\title{
Early Physics Reach of the ATLAS Detector
}

\author{
Ivor Fleck \\ On behalf of the ATLAS Collaboration \\ Universität Freiburg, Physikalisches Institut, Hermann-Herder Str.3, D-79104 Freiburg, \\ Germany \\ e-mail: Ivor.Fleck@cern.ch \\ Received:
}

\begin{abstract}
The first year of data taking at ATLAS should be a very challenging and rewarding time. It will include commissioning of the detector and many interesting physics results. The commissioning of the detector components will mainly rely on physics signals. The staging of some subdetectors will influence the B physics program and result in slightly reduced significances in most physics analyses. The searches for Higgs bosons and supersymmetric particles have been studied in great detail. If a Higgs boson from the Standard Model exists, it will be discovered within the first year. If supersymmetry exists the discovery of at least one light Higgs boson as well as some supersymmetric particles is very probable within the first year of the LHC.
\end{abstract}

PACS: 25.70.Ef; 21.60.Gx; 27.30.+t

\section{Introduction}

The aim of this paper is to show what we can reach with one year of data taking with the ATLAS detector at the LHC, how we are going to achieve it and how the results will compare to the results from other collider experiments at that point in time.

First an overview is given about the physics results expected from other collider experiments by the year 2007, then the commissioning of some detector components is described. Afterwards the staging of some detector components is discussed. Finally, two examples of physics results expected from one year of data taking at low luminosity, corresponding to $2 \times 10^{33} \mathrm{~cm}^{-2} \mathrm{~s}^{-1}$ are shown shown. Most results presented here are taken from the ATLAS Technical Design Report [1].

When data taking with the ATLAS detector at the LHC starts in the year 2007 the most precise existing measurements of variables also accessible at the LHC will stem from several sources. The expected values are listed in Table 1.

All results presented here correspond to an integrated luminosity of $10 \mathrm{fb}^{-1}$, which will be taken in approximately one year of LHC operation at initial luminosity. The event rates expected for this luminosity are given in Table 2. 


\begin{tabular}{|l|l|l|}
\hline Variable & Precision & Experiment \\
\hline W boson mass & 20 to $30 \mathrm{MeV}$ & LEP + Tevatron \\
Top quark mass & $2 \mathrm{GeV}$ & Tevatron \\
Structure functions & few percent up to $Q^{2}=10^{4} \mathrm{GeV}^{2}$ & HERA \\
Higgs boson & $\begin{array}{l}\text { possible exclusion up to } 175 \mathrm{GeV} \\
\text { discovery unlikely }\end{array}$ & Tevatron \\
\hline
\end{tabular}

Table 1. Precision of variables expected in the year 2007.

\begin{tabular}{|l|l|l|l|}
\hline Process & Events/s & $\begin{array}{l}\text { Events for } \\
10 \mathrm{fb}^{-1}\end{array}$ & $\begin{array}{l}\text { total statistics collected } \\
\text { at previous machines } \\
\text { by 2007 }\end{array}$ \\
\hline $\mathrm{W} \rightarrow \mathrm{e} \nu$ & 30 & $10^{8}$ & $10^{4}$ LEP, $10^{7}$ Tevatron \\
$\mathrm{Z} \rightarrow \mathrm{ee}$ & 3 & $10^{7}$ & $10^{7}$ LEP \\
$t \bar{t}$ & 2 & $10^{7}$ & $10^{4}$ Tevatron \\
$b \bar{b}$ & $10^{6}$ & $10^{12}-10^{13}$ & $10^{9}$ Belle, BaBar \\
$\mathrm{SM} \mathrm{Higgs}(\mathrm{m}=130 \mathrm{GeV})$ & 0.04 & $10^{5}$ & $?$ \\
$\tilde{g} \tilde{g}(\mathrm{~m}=1 \mathrm{TeV})$ & 0.002 & $10^{4}$ & - \\
\hline
\end{tabular}

Table 2. Event rates and the total number of events expected in the first year of data taking with the ATLAS detector. Also given is the total number of events for the same Standard Model (SM) and Supersymmetry (SUSY) processes expected from other experiments up to the year 2007.

\section{Commissioning}

The precision to which the detector needs to be understood is given by the desired precision of the physics results. For example in order to achieve an equal error on the indirect determination of the Higgs mass from the measurements of the top quark and $\mathrm{W}$ boson masses, the uncertainty on the mass of the $\mathrm{W}$ has to be a factor of 150 times smaller than that of the top. With an expected uncertainty on the top mass of about $2 \mathrm{GeV}$ the uncertainty on the $\mathrm{W}$ mass should therefore be about $15 \mathrm{MeV}$. This requires a determination of the lepton (electron and muon) absolute energy and momentum scale to $0.02 \%$, putting severe constraints on the understanding of the detector components.

To reach the required level of understanding of the detector a very thorough commissioning has to be performed. The commissioning of the detector components is done in several steps:

- performance studies and calibration in test beams

- mapping of detector material

- measurement of the alignment between detector parts

- calibration of the read-out electronics 
- cosmic running

- one proton beam in the LHC

- proton proton collisions

Many of the detector components will have been tested with test beams or cosmic rays for their response, alignment and uniformity before they are installed in the ATLAS detector. Once the ATLAS detector is assembled the first major step for commissioning is taking cosmic data. Then the LHC machine will turn on with only one proton beam. This operation mode will be used to check the readout chain of the detector components. Finally, the most important step in the commissioning is analyzing the data from proton proton collisions. The very high rates of well known particles, like the $\mathrm{Z}$ boson, allow for e.g. calibration of the detector response. Particles like muons will be used for the alignment.

\subsection{Inner Detector}

The Inner Detector (ID), consisting of three layers of silicon Pixel detectors, four double layers of silicon strips (SCT) and the transition radiation tracker, should be aligned such that the degradation of the track parameter resolution stemming from misalignment is less than $20 \%$. This leads to the requirement that in $\mathrm{R} \phi$ the Pixel detectors should be aligned to $\sim 7 \mu \mathrm{m}$ and the SCT detectors to $\sim 12 \mu \mathrm{m}$.

The alignment of the Inner Detector will start with the Pixels and the SCT, together called the Precision Tracker. Best suited for the alignment are tracks from muons. Stiff tracks crossing the whole ID are needed. Single muon production from the decay $\mathrm{W} \rightarrow \mu \nu$ occurs with a rate of about $3 \mathrm{~Hz}$ and for muons with $p_{T}>6 \mathrm{GeV}$ from b decays with a rate of about $50 \mathrm{~Hz}$. The residuals between the track fit and the reconstructed hit position on each silicon plane are found by fitting the tracks in the rest of the Precision Tracker, but excluding that plane.

Figure 1 shows the expected width of the residuals in the barrel layers of the Precision Tracker. The width of the residuals from muon tracks from W decays is about $20 \mu \mathrm{m}$ for the barrel part of the Pixels and about $40 \mu \mathrm{m}$ for the barrel part of the SCT. Using an integrated luminosity of about $0.1 \mathrm{fb}^{-1}$, which could be accumulated within only one day of data taking at low luminosity, the precision of the alignment will be $1 \mu \mathrm{m}$ for the Pixels and less than $2 \mu \mathrm{m}$ for the SCT.

\subsection{Calorimeter}

The calorimeter of the ATLAS detector is divided into two parts, the electromagnetic and the hadronic calorimeter.

For the reconstruction of the energy in the electromagnetic calorimeter there are 448 regions covering an area in pseudo rapidity and azimuthal angle $\Delta \eta \times$ $\Delta \phi=0.2 \times 0.4$. For each of these regions, several effects contribute to a local constant term $\left(c_{L}\right)$ of around $0.5 \%$. The main contributions arise from uncertainties in the geometry (e.g. residual Accordion modulation), mechanical thickness of the absorber and gaps and the uniformity of the calibration amplitude. Test 

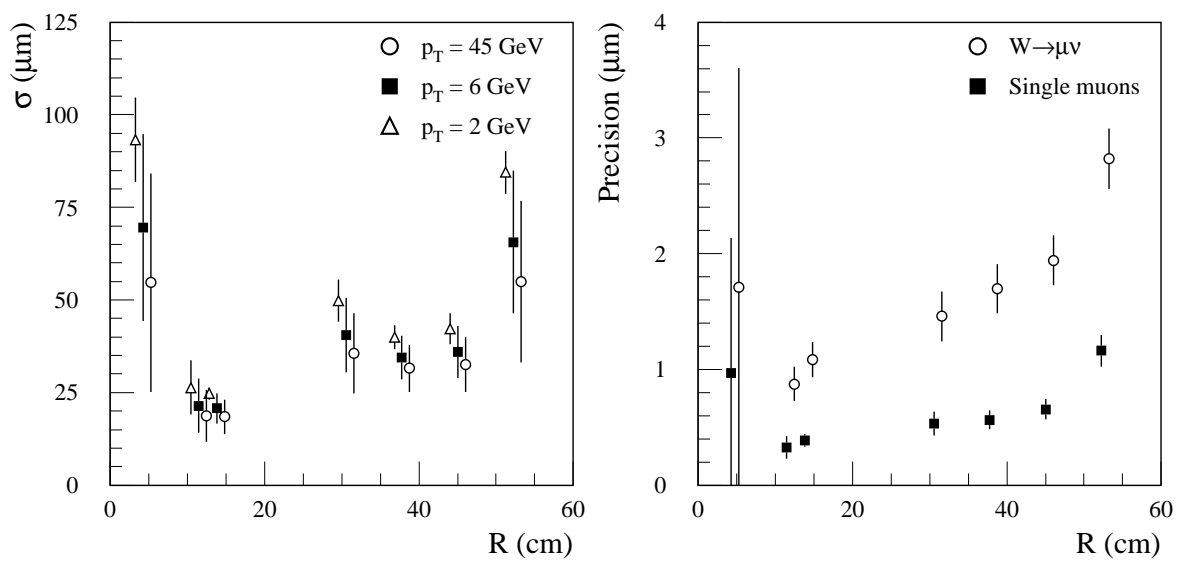

Fig. 1. Precision of the $\mathrm{R} \phi$ alignment of the barrel modules achieved in the different layers of the pixel detectors (3 layers below $20 \mathrm{~cm}$ ) and the layers of the SCT (4 layers above $30 \mathrm{~cm}$ ). The "error bars" indicate the spread of measurements from different modules in the same layer. The points from the $\mathrm{W}$ decay and the single muons are horizontally offset to avoid overlap.

beam results of 4 out of 32 barrel modules and 3 out of 16 end cap modules show that the desired uniformity of around $0.5 \%$ is reached. In addition to the local constant term, the uncertainty in the energy calibration (long range nonuniformities $c_{L R}$ ) has to be controlled within $0.4 \%$ locally. This calibration will first be done using test beams, resulting in a knowledge of the energy scale to $1-2 \%$. As this is well above the desired final uncertainty, a calibration using $\mathrm{Z}$ $\rightarrow \mathrm{e}^{+} \mathrm{e}^{-}$events from proton proton collisions will be made. These events can be used to make a stand alone calibration, imposing a $\mathrm{Z}$ mass constraint and using only the electromagnetic calorimeter. This channel has a large rate of about $1 \mathrm{~Hz}$ and is almost background free. The mass of the $\mathrm{Z}$ boson is close to the mass of the $\mathrm{W}$ and the Higgs, and errors from non-linearities in the calorimeter response for particles with different energies will therefore be small in the reconstruction of the masses of these two particles. Within only a few days of data taking, more than 250 events will be taken per region for $|\eta|<2.5$. This is sufficient for the intercalibration of all 400 regions with a precision better than $0.4 \%$. The difference between the correct and the reconstructed calorimeter coefficients from a Monte Carlo study is shown in Figure 2. The total constant term of the calorimeter will therefore be less than $0.7 \%$, which is the required uncertainty to observe the mass peak of the Higgs in the decay $\mathrm{H} \rightarrow \gamma \gamma$ above the huge background in the first year.

For the hadronic calorimeter, the absolute energy scale for hadronic jets will be known to $5-10 \%$ from test beams and Monte Carlo studies. To achieve a better resolution, an in-situ calibration will be performed. One important physics sample will be the hadronic decay of $\mathrm{W}$ bosons, $\mathrm{W} \rightarrow$ jet jet. As non-linearities in the energy scale cause problems for jets with higher energies, an additional sample, events with a jet recoiling against a $\mathrm{Z}$ boson, will be used. 

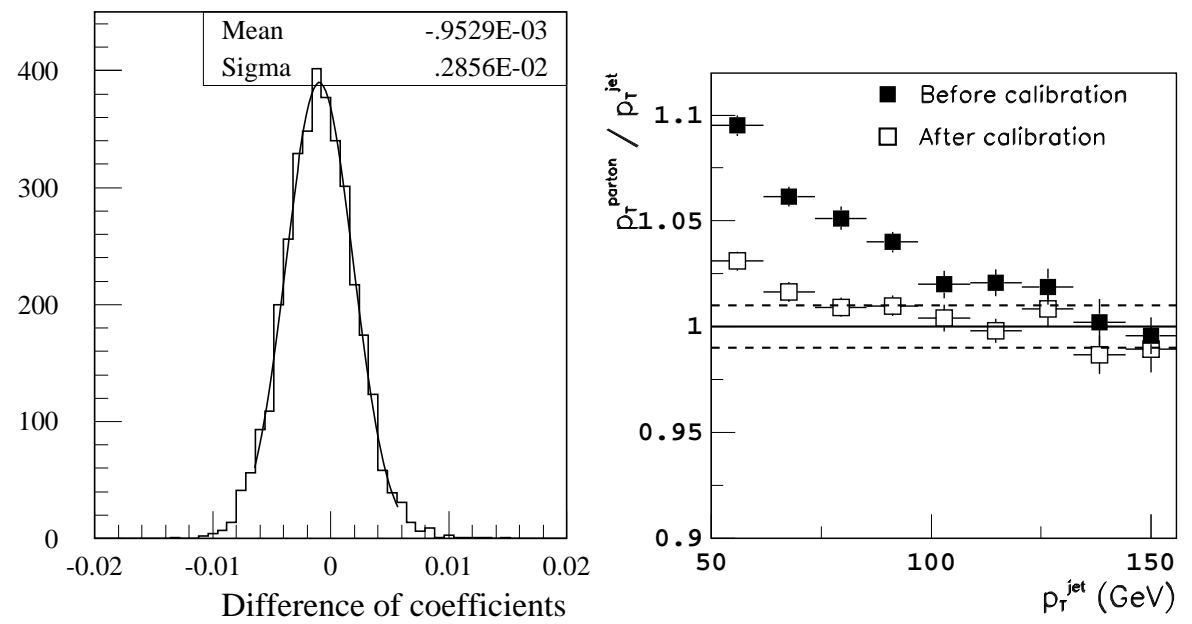

Fig. 2. Difference of EM calorimeter coefficients after reconstruction using $\mathrm{Z} \rightarrow$ $\mathrm{e}^{+} \mathrm{e}^{-}$events between correct and reconstructed coefficients (left Figure). Resolution of the hadronic calorimeter before and after the calibration using physics events (right Figure).

For the calibration with $\mathrm{W}$ bosons, events from the process $\mathrm{tt} \rightarrow \mathrm{WbWb} \rightarrow$ $(\ell \nu \mathrm{b})$ (jet jet $\mathrm{b})$ will be used. With the identification of the two $\mathrm{b}$ jets, the two remaining jets form a clean sample for the reconstruction of the $\mathrm{W}$ mass. A clean sample of $45 \mathrm{k}$ events should be collected in the first year, resulting in a resolution in the transverse momentum $p_{T}$ of $1 \%$ in the range between 50 and $250 \mathrm{GeV}$. The improvement in the resolution of the transverse momentum is shown in Figure 2. New studies, not included in the Figure, have been performed and result in the resolution quoted above.

\subsection{Muon Detector}

The muon detector consists of monitored drift tubes and cathode strip chambers, together referred to as Precision Chambers, for the detection of muons and the measurements of their momentum. Thin gap chambers and resistive plate chambers are used for triggering and for sending a timing signal. The muon detector is inside a toroidal magnetic field with a strength of up to 4 Tesla.

The momentum resolution and the absolute calibration of the muon system depend strongly on the alignment of the precision chambers, the knowledge of the magnetic field and the knowledge of the muon energy loss inside the calorimeters. To achieve the best knowledge of these properties the following calibration procedures will be used.

For the alignment, special runs with the toroidal magnetic field off and the solenoid field on will be taken. This will lead to straight tracks from muons inside the muon system with a well known momentum measured with the Inner Detector. The knowledge of the track momentum from the Inner Detector allows muons with transverse momenta $p_{T}>10 \mathrm{GeV}$ to be selected, to minimize effects 
from multiple scattering. These tracks will be used to align the muon chambers with respect to each other and with respect to the additional muon alignment system. For a run only one hour long the sagitta correction in the barrel system will be known with a precision of less than $30 \mu \mathrm{m}$. This is well below the intrinsic chamber error of $50 \mu \mathrm{m}$. With the toroidal magnetic field on, the position of the chambers and the alignment system may change by a few $\mathrm{mm}$ with respect to the position with no field. The alignment system is capable of measuring the changes in its own position as well as of the position in the precision chambers. Therefore the same precision in the sagitta correction is expected with the toroidal magnetic field on.

For the measurement of the toroidal magnetic field 5000 Hall probes will be used. They will determine the field strength with a relative precision of $0.1 \%$. This precision is above the intended final precision of $0.02 \%$. A calibration of the magnetic field using physics events will therefore be made. The best process is $\mathrm{Z} \rightarrow \mu \mu$, which with a rate of 30000 events per day delivers enough events. Requiring the invariant mass of the muon pair to be equal to the $\mathrm{Z}$ mass, the magnetic field can be determined locally in principle. But there is one difficulty: the energy loss of the muons inside the calorimeters. This energy loss is about $4 \mathrm{GeV}$, depending on the thickness of the calorimeter transversed and on the muon momentum. The material of the calorimeter and the relative muon energy loss are not known well enough to correct for this effect with the desired precision. The energy loss in the calorimeter would have to be known with an accuracy of $10 \mathrm{MeV}$ to reach the ultimate goal of $0.02 \%$ in momentum resolution. It is therefore necessary to perform a simultaneous fit to the magnetic field and the energy loss of the muons using the constraint of the $\mathrm{Z}$ mass, taking into account the finite width of the $\mathrm{Z}$. This fit will have to be made in many regions of $(\eta, \phi)$ and the outcome will depend on how many regions can be fitted. To reach the final goal of $0.02 \%$ on the muon momentum resolution in the first year will therefore be challenging.

\subsection{Track resolution}

For the total track resolution, several detector components will work together. Nonetheless, the main information will come from the Inner Detector. The measured track momentum in the ID is subject to bremsstrahlung effects. The distribution of the material in the ID will have to be understood to $1 \%$ to reach a final track accuracy of $0.02 \%$. This will again be done using physics events, as even a detailed survey of the ID before the installation cannot achieve such a precision. It is planned to use electrons from $\mathrm{W}$ decays to measure the distribution of the ratio of the energy measured in the electromagnetic calorimeter to the track momentum. This distribution of $\mathrm{E} / \mathrm{p}$ is not only sensitive to the total amount of material inside the ID, but also to the radial distribution of the material. The data of a few weeks will be sufficient to achieve the needed precision.

Another important parameter for the track momentum determination is a precise knowledge of the magnetic field of the solenoid in the ID. Before the installation of the ID the field will be mapped using Hall probes with a precision of $0.05 \%$. As this is not sufficient, tracks will be used to achieve the final precision 

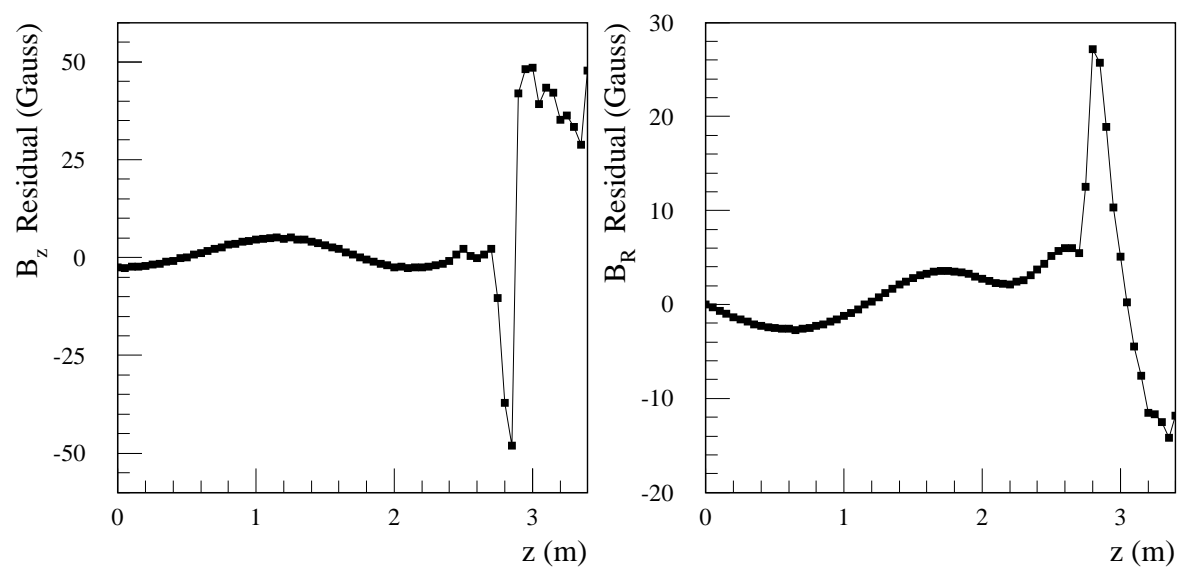

Fig. 3. Difference between the simulated and reconstructed magnetic field from a Monte Carlo study for the axial (left) and radial (right) components of the solenoid at a radius $R=75 \mathrm{~cm}$

of $0.02 \%$. To better understand the strength of the magnetic field, pairs of tracks from resonance decays like the $J / \psi$ or the $\mathbf{Z}$ boson will be used. They can be used in a fit to the global field strength, resulting in residuals between the simulated and the fitted field of less than 4 Gauss, i.e. better than $0.02 \%$, over the whole length of the solenoid. The distribution of the residuals as a function of the $z$ position is shown in Figure 3. A larger deviation is only observed towards the end of the solenoid at $|z|=2.65 \mathrm{~m}$.

\subsection{Implications of Staging}

Due to limited availability of resources and schedule constraints, the completion of some detector components will be deferred by a couple of years.

The detector components that are staged are:

- The middle layer of the pixel detector,

- the outer end-cap of the transition radiation detector,

- the scintillator in the gap between the barrel and the end-cap calorimeters,

- the transition chambers (EES/EEL) of the precision muon chambers,

- part of the liquid argon calorimeter ROD (read out driver) system, and

- a large part of the high level trigger (HLT) and data acquisition.

The guiding principle for the choice of these detector components is that all detector components needed for having good physics results in the first year be present.

Several studies have been performed to evaluate the implications of the staging of the components listed above. For example, the staging of the middle pixel 
layer will reduce the rejection power against light quarks in a b quark sample. When requiring a $60 \%$ b quark efficiency, the u-quark rejection is degraded by $30 \%$ when using two pixel layers instead of three. Applying this degraded rejection to the Higgs channel $\mathrm{ttH} \rightarrow \mathrm{tt} \mathrm{bb}$, the significance of this channel is reduced by $8 \%$. To compensate for the missing pixel layer and to achieve the same significance, $15 \%$ more integrated luminosity is needed. Similar losses in significance are expected for the process $\mathrm{H} \rightarrow 4 \mathrm{e}$ due to the missing gap scintillator and for the process $\mathrm{A} / \mathrm{H} \rightarrow \mu \mu$ from the staging of the muon detector.

A more severe degradation of the detector performance arises from the staging of the trigger electronics. The approach for the ATLAS trigger is to make mostly inclusive selections with low $p_{T}$ thresholds for fundamental objects like leptons. Ideally one would like to keep a safety margin in the trigger rate, possibly greater than a factor of 2, for uncertainties for example from QCD cross-sections. Some bandwidth should also be reserved for control triggers, for example for calibration.

The implication of the staging of the HLT is that the output bandwidth from the level 1 triggers has been reduced from $75 \mathrm{kHz}$ to $25 \mathrm{kHz}$. As a result, the trigger threshold must be raised, in particular jeopardizing b quark physics. As a consequence there will be no dedicated B physics triggers in the first year. In addition there will be no safety margin for high $p_{T}$. The rise in the trigger thresholds also results in smaller event samples for decays with large contributions below the new trigger thresholds. For example for the decay ttH $\rightarrow$ ttbb with one $\mathrm{W}$ from the top quark decay decaying leptonically the significance will be reduced by $8 \%$ when raising the trigger thresholds for leptons (electrons and muons) from 20 to $30 \mathrm{GeV}$. This reduction in significance is in addition to that arising from the missing pixel layer.

The overall impact of the staging is that for many analyses 10-15\% more integrated luminosity will be needed to achieve the same significance as with the full detector. In addition there will be no dedicated B physics program in the first year. For operation with high luminosity, the fully instrumented detector is therefore needed.

\section{Physics results in the first year}

In this section, two of the most important physics topics that will be addressed during the first year of data taking are presented. An integrated luminosity of $10 \mathrm{fb}^{-1}$ is assumed.

Before any analysis can be done, the QCD processes have to be understood with great precision, as they represent the major background to most analyses. The production cross-sections for most processes are also controlled by QCD. It is therefore mandatory to measure first the parton distribution functions (pdf). From HERA, the pdfs will be known with a precision of a few percent for values of $Q^{2}$ up to $10^{4} \mathrm{GeV}^{2}$ [5]. Further knowledge will come from the Tevatron experiments. The measurement of the pdfs at the LHC in the overlap regions with the previous experiments is the first step to verify the measurement procedures. In the next step, the known pdfs will be evolved in $Q^{2}$ using the DGLAP evolution equations. From the uncertainty in the knowledge of $\alpha_{s}$, the evolution 


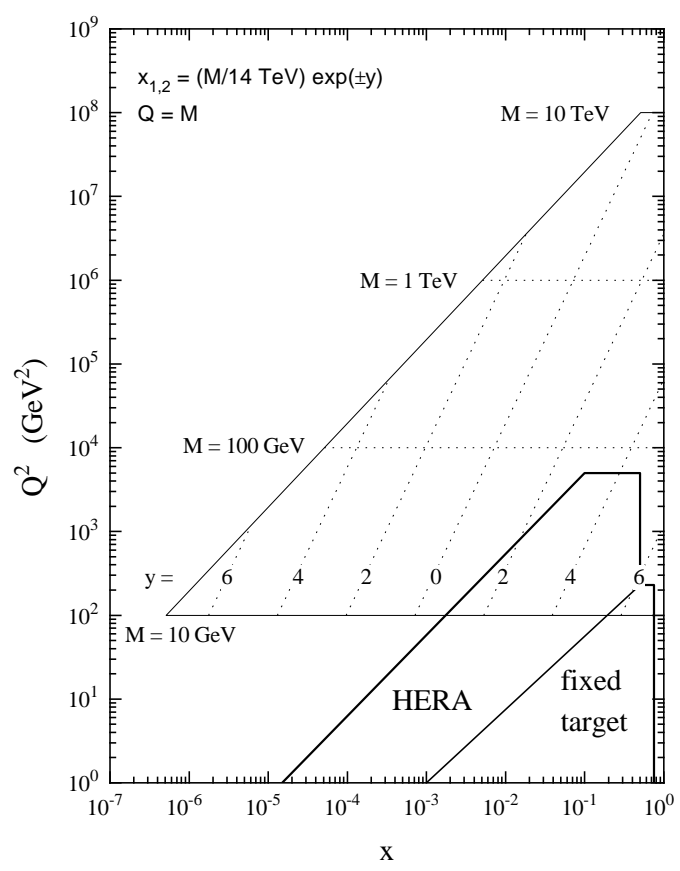

Fig. 4. Reach in $x$ and $Q^{2}$ at the LHC. The dotted horizontal lines correspond to the production of a particle with mass $M$, while the other dotted lines correspond to the rapidity $y$.

in $Q^{2}$ will lead to uncertainties in the structure functions of several percent. For the measurement of the pdfs at ATLAS, Drell-Yan processes can be used for the quark distribution functions and direct photon and jet production for the gluon distribution functions.

For QCD processes at the LHC, the knowledge of the gluon distribution functions is most important, as most processes are gluon induced. To achieve the best knowledge of the pdfs, a global fit to the previous and the new data is necessary. It is also important to assess the error on the calculation of the pdfs, as this allows the only realistic estimate of the expected uncertainty in all involved processes, such as the cross-section for new physics processes or the background calculation for Standard Model measurements.

The region in $x$ and $Q^{2}$ accessible at the LHC and the overlap with HERA is shown in Figure 4. As can be seen there is a small overlap region with HERA, and the reach of the Tevatron experiments is up to $Q^{2}=10^{6} \mathrm{GeV}^{2}$. A large new region will nevertheless be explored.

\subsection{Higgs}

The most searched for particle is the Higgs boson. It has eluded discovery so far, but it is due to be discovered at the LHC, if it exists. For the Higgs boson 
of the Standard Model there is an upper limit on its mass of about $1 \mathrm{TeV}$ from unitarity arguments [2]. Direct searches for the Higgs boson at LEP [3] exclude a mass below $114.4 \mathrm{GeV}$. This is well above the current most probable value of $91 \mathrm{GeV}$ derived from precision measurements of electroweak observables [4]. Due to the logarithmic dependence of the electroweak variables on the Higgs mass, the upper limit on the mass is $211 \mathrm{GeV}$ at $95 \%$ confidence limit. It is therefore not guaranteed that the Higgs boson will be discovered at the Tevatron, which is sensitive up to $130 \mathrm{GeV}$ [6].

For ATLAS, studies have been performed for discovering the Higgs with masses between the current direct lower limit and the maximum allowed mass of $1 \mathrm{TeV}$. The expected signal significance is shown in Figure 5.

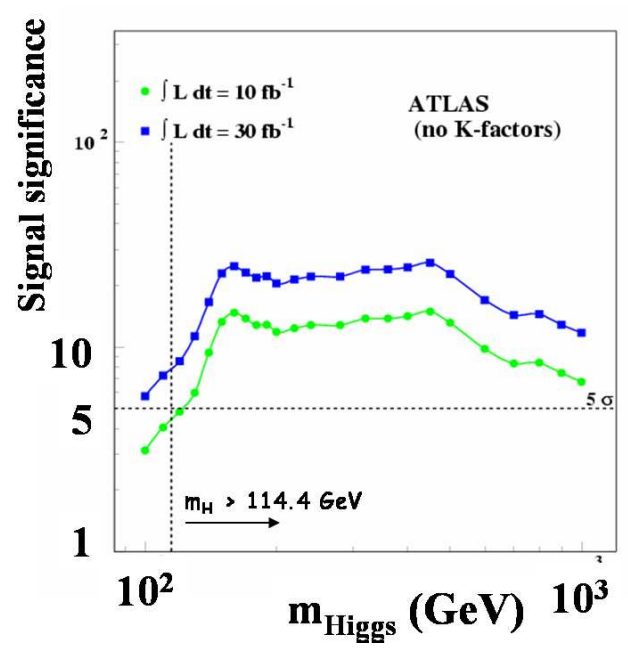

Fig. 5. Signal significance for a Higgs discovery for integrated luminosities of $10 \mathrm{pb}^{-1}$ (lower line) and $30 \mathrm{pb}^{-1}$ (upper line). Also shown is the lower mass limit on the Higgs mass from LEP.

In the region of Higgs masses between 200 and $600 \mathrm{GeV}$ the decay $\mathrm{H} \rightarrow$ $\mathrm{ZZ} \rightarrow \ell^{+} \ell^{-} \ell^{\prime+} \ell^{\prime-}$ with $\ell=e, \mu$ is best suited to discover the Higgs. The background rate for this final state is smaller than the signal and the width of the Higgs is larger than the experimental resolution, at least for Higgs masses above $300 \mathrm{GeV}$. A discovery, i.e. a signal with a significance greater than 5 standard deviations, is achievable with the data in the first year. The signal for a Higgs with a mass of $300 \mathrm{GeV}$ is shown in Figure 6. The signal significance can be increased by requiring the transverse momentum of the harder of the two $\mathrm{Z}$ bosons, $p_{T}^{\max }\left(\mathrm{Z}_{1}, \mathrm{Z}_{2}\right)$ to be larger than a given cut value. In Figure 5 a cut value of $100 \mathrm{GeV}$ has been applied. For an integrated luminosity of $10 \mathrm{fb}^{-1}$ the signal lies well above the background. Once the signal has been established in the four lepton channel a confirmation in the channel $\mathrm{H} \rightarrow \mathrm{ZZ} \rightarrow \ell^{+} \ell^{-}$jet jet is possible.

For Higgs masses above $600 \mathrm{GeV}$ the four lepton channel is statistically limited. Other decay modes, like $\mathrm{H} \rightarrow \mathrm{ZZ} \rightarrow \ell^{+} \ell^{-} \nu \bar{\nu}$, or $\mathrm{H} \rightarrow \mathrm{WW} \rightarrow \ell \nu$ jet jet which has a branching ratio 150 times larger, will be deployed. This channel 

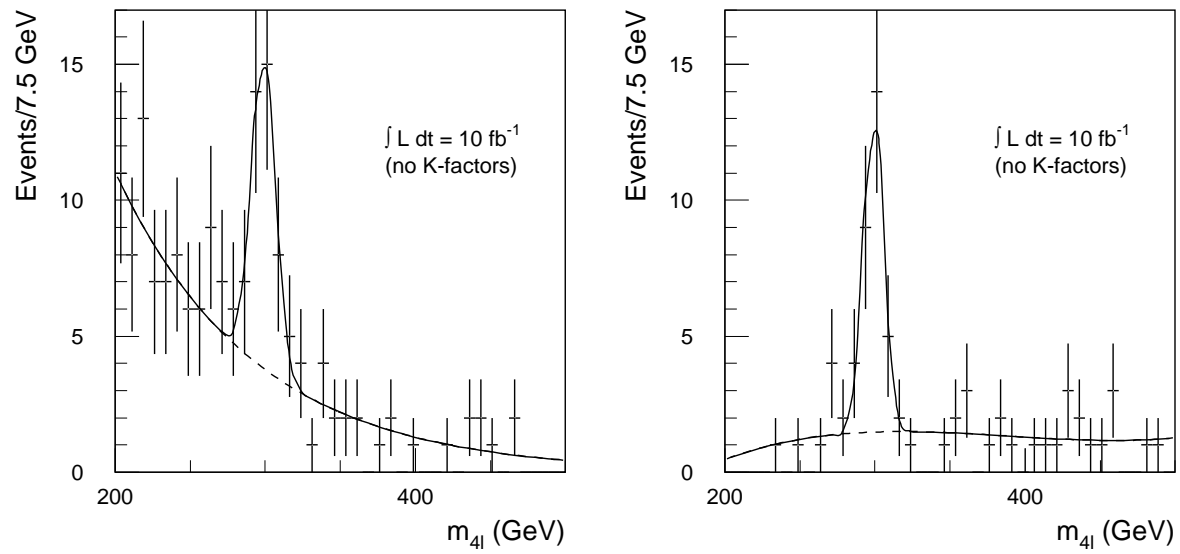

Fig. 6. Invariant mass of four leptons before (left) and after (right) applying a cut on the transverse momentum of the harder $\mathrm{Z}$ boson, $p_{T}^{\max }\left(\mathrm{Z}_{1}, \mathrm{Z}_{2}\right)$. For the Higgs signal $m_{H}=300 \mathrm{GeV}$ has been used.

has a much larger background than the four lepton channel but due to its much larger branching ratio it can contribute to the discovery. In the large mass region, the main contribution is from channels with missing transverse momentum and jets. A very good measurement of the jet energies is therefore essential.

When combining the significances from all the above channels a Higgs boson with a mass above $200 \mathrm{GeV}$ can be discovered within the first year.

For masses below $200 \mathrm{GeV}$ the Higgs is more difficult to discover, especially for masses close to the experimental limit of $114.4 \mathrm{GeV}$. Several channels are needed to discover a Higgs for an integrated luminosity of $10 \mathrm{fb}^{-1}$. The traditional channel considered for Higgs discovery is $\mathrm{H} \rightarrow \gamma \gamma$. It is best suited for use in the first year, as this channel relies on the electromagnetic calorimeter only. There is no direct coupling between the Higgs and the photon, so this process has to proceed via loops and the branching ratio is therefore very small, being only $0.2 \%$ for Higgs masses around $120 \mathrm{GeV}$. To reconstruct a clean Higgs mass peak above the background, a constant calorimeter term below $0.7 \%$ is needed. With this condition fulfilled, a significance of 2 can be achieved from this channel for the first year.

However, other channels contributing with similar significance are the associate Higgs production $\mathrm{ttH} \rightarrow \mathrm{ttbb}$ and the vector boson fusion $\mathrm{qqH} \rightarrow \mathrm{qq} \tau \tau$. The number of signal and background events expected during the first year are given in Table 3.

The observation of all three channels is important to extract a convincing signal in the first year. The systematic uncertainties for the three channels are very different; they have different production mechanisms, different decay modes and rely on different parts of the detector. For the associated ttH production, $b$ tagging is essential, as all four b quarks, two from the Higgs decay and one from each top decay, have to be identified to efficiently suppress the background. For the vector boson fusion, recent studies [7] showed that this channel may already 


\begin{tabular}{|l|l|l|l|}
\hline process & $\mathrm{H} \rightarrow \gamma \gamma$ & $\mathrm{ttH} \rightarrow \mathrm{ttbb}$ & $\mathrm{qqH} \rightarrow \mathrm{qq} \tau \tau$ \\
\hline Signal $(\mathrm{S})$ & 130 & 15 & $\sim 10$ \\
Background $(\mathrm{B})$ & 4300 & 45 & $\sim 10$ \\
$\mathrm{~S} / \sqrt{B}$ & 2.0 & 2.2 & $\sim 2.7$ \\
\hline
\end{tabular}

Table 3. Higgs decay channels and number of signal and background events for an integrated luminosity of $10 \mathrm{pb}^{-1}$.

be accessible in the low mass region in the first year. Here jet tagging up to the very forward region $(|\eta| \approx 5)$ is needed. The expected significance from the vector boson fusion channels is shown in Figure 7 .

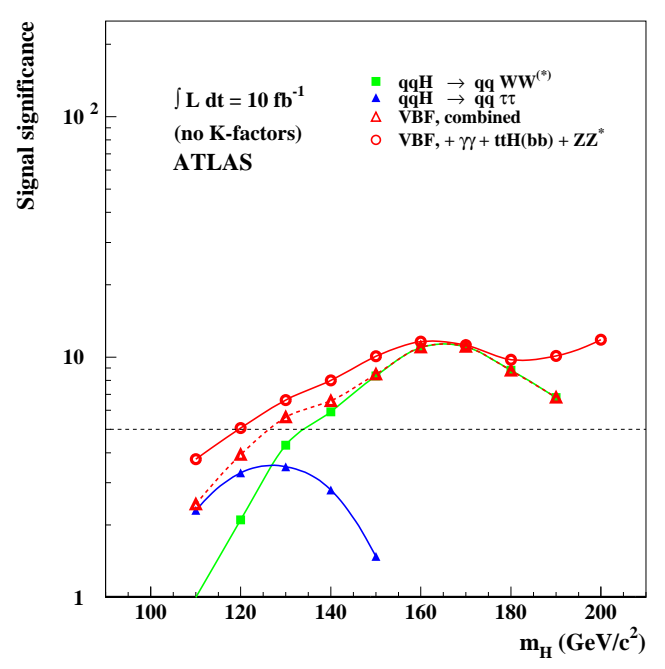

Fig. 7. Expected significance for the vector boson fusion channels for Higgs masses below $200 \mathrm{GeV}$.

The results presented here are all conservative, as only very simple analyses have been used and no K-factors have been included. The Higgs boson can therefore be observed in the first year with a significance above $5 \sigma$ over the whole mass range between the current lower limit from LEP and the theoretical upper limit of $1 \mathrm{TeV}$.

In theories beyond the Standard Model, more than one Higgs boson may exist. The most favoured theory, supersymmetry (MSSM), predicts five Higgs bosons: $\mathrm{h}, \mathrm{H}, \mathrm{H}^{+}, \mathrm{H}^{-}$, A. The mass of the lightest neutral Higgs, the $\mathrm{h}$, has to be below $135 \mathrm{GeV}$. The branching ratios of the decay modes of these Higgs bosons depend on the parameters of the MSSM. Decay modes that have no significance in the search for a Standard Model Higgs can become important. One such channel is $\mathrm{H} / \mathrm{A} \rightarrow \mu^{+} \mu^{-}$, shown in Figure 8 , which has a large sensitivity in parameter regions that are not already excluded from LEP and are not covered 
by the searches for the Standard Model Higgs. The muon spectrometer is crucial for covering this part of the parameter space.

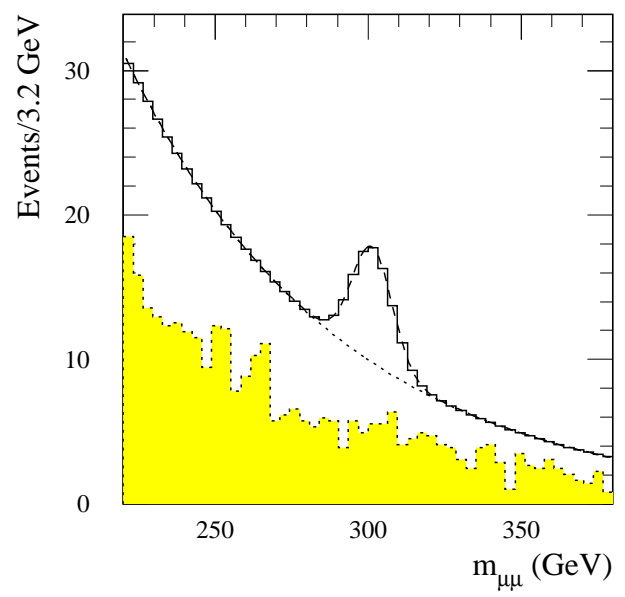

Fig. 8. Invariant mass of a $\mu \mu$ pair from a SUSY Higgs with a mass of $300 \mathrm{GeV}$. The shaded histogram shows the reducible $t \bar{t}$ background, the dashed curve the total background and the solid line the sum of Higgs signal and background. Note that this plot is for an integrated luminosity of $30 \mathrm{pb}^{-1}$.

This parameter space is also covered by final states consisting of a tau pair, but the identification of taus will be more difficult in the first year than the identification of muons. The invariant mass of a tau pair is shown in Figure 9 for different Higgs masses.

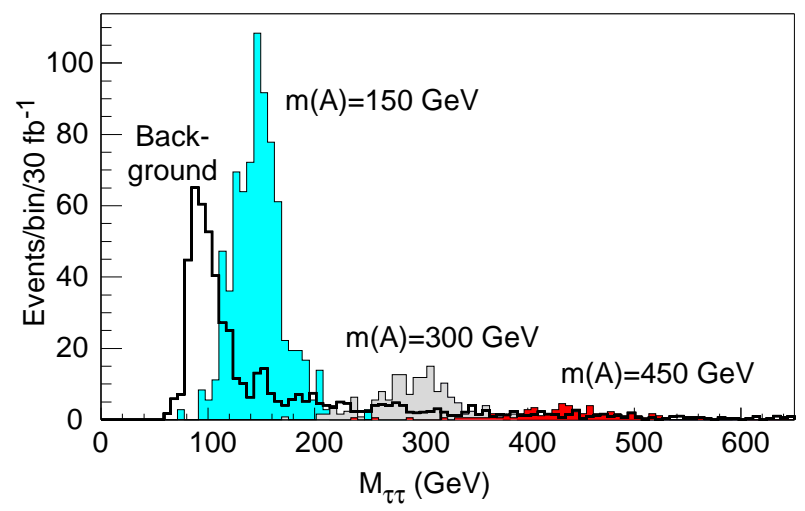

Fig. 9. Invariant mass of a $\tau \tau$ pair from a SUSY Higgs for three different Higgs masses. Note that this plot is for an integrated luminosity of $30 \mathrm{pb}^{-1}$.

In the first year nearly the whole MSSM parameter space is already accessible and a discovery of a supersymmetric Higgs most probable. 


\subsection{Supersymmetry}

At the LHC, SUSY particles, especially squarks and gluinos, can be produced with very large rates. The cross-sections for gluino or squark pair production can be as high as several $100 \mathrm{fb}$ for masses above $1 \mathrm{TeV}$. These large cross-sections stem from the large gluon content inside the protons and the strong coupling which also applies to coloured SUSY particles.

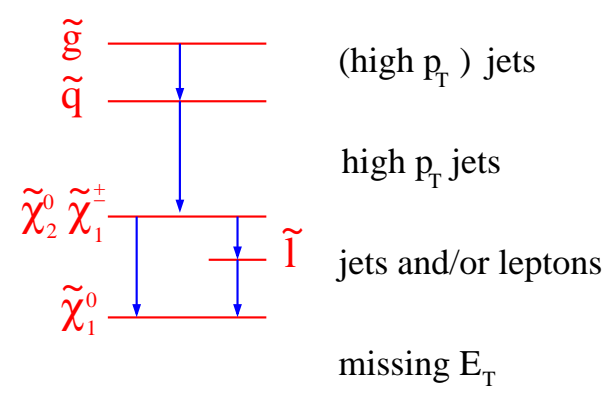

Fig. 10. Decay chain of gluinos.

The decay chain of squarks or gluinos, as shown in Figure 10, leads to jets with high transverse momentum due to the large mass difference between the squarks (or gluinos) and the charginos or neutralinos. In addition, large missing transverse energy is expected due to the presence of lightest neutralinos (LSP) in the final state. For some decay modes isolated leptons are also present.

These event signatures can be used to effectively separate the SUSY signal from the background. Squarks or gluinos with masses up to $1.5 \mathrm{TeV}$ could be discovered with an integrated luminosity of only $0.1 \mathrm{fb}^{-1}$, corresponding to only one day of data taking. Within the first year it is possible to discover squarks or gluinos with masses up to $2.3 \mathrm{TeV}$. The exact numbers depend on the values of the SUSY parameters. The discovery curves for $\tan \beta=10, \mu>0$ and $A_{0}=0$ are shown in the $M_{0}-M_{1 / 2}$ plane in Figure 11.

Just looking for an excess of events with large missing transverse energy and with jets with large transverse momentum will be a hint of the presence of SUSY, but only by measuring the masses of the SUSY particles and the overall SUSY mass scale can a more believable sign of SUSY be established.

Some mass information can be obtained from decay chains. The two body decay chain $\tilde{\chi}_{2}^{0} \rightarrow \tilde{\ell}^{ \pm} \ell^{\mp} \rightarrow \ell^{ \pm} \tilde{\chi}_{1}^{0} \ell^{\mp}$ leads to a very sharp edge in the distribution of the invariant mass of the two leptons. The position of the edge depends on the mass differences between the $\tilde{\chi}_{2}^{0}$ and the slepton $(\tilde{\ell})$ and on the mass difference between the slepton and the lightest neutralino $\left(\tilde{\chi}_{1}^{0}\right)$.

$$
\max \left(m\left(\ell^{+} \ell^{-}\right)\right)=m\left(\tilde{\chi}_{2}^{0}\right) \sqrt{1-\frac{m^{2}(\tilde{\ell})}{m^{2}\left(\tilde{\chi}_{2}^{0}\right)}} \sqrt{1-\frac{m^{2}\left(\tilde{\chi}_{1}^{0}\right)}{m^{2}(\tilde{\ell})}}
$$

The very sharp edge in this distribution, after subtracting background determined from like sign lepton pairs, is shown in Figure 12. Note that the integrated luminosity used to produce this plot corresponds to $100 \mathrm{fb}^{-1}$. Nevertheless for 


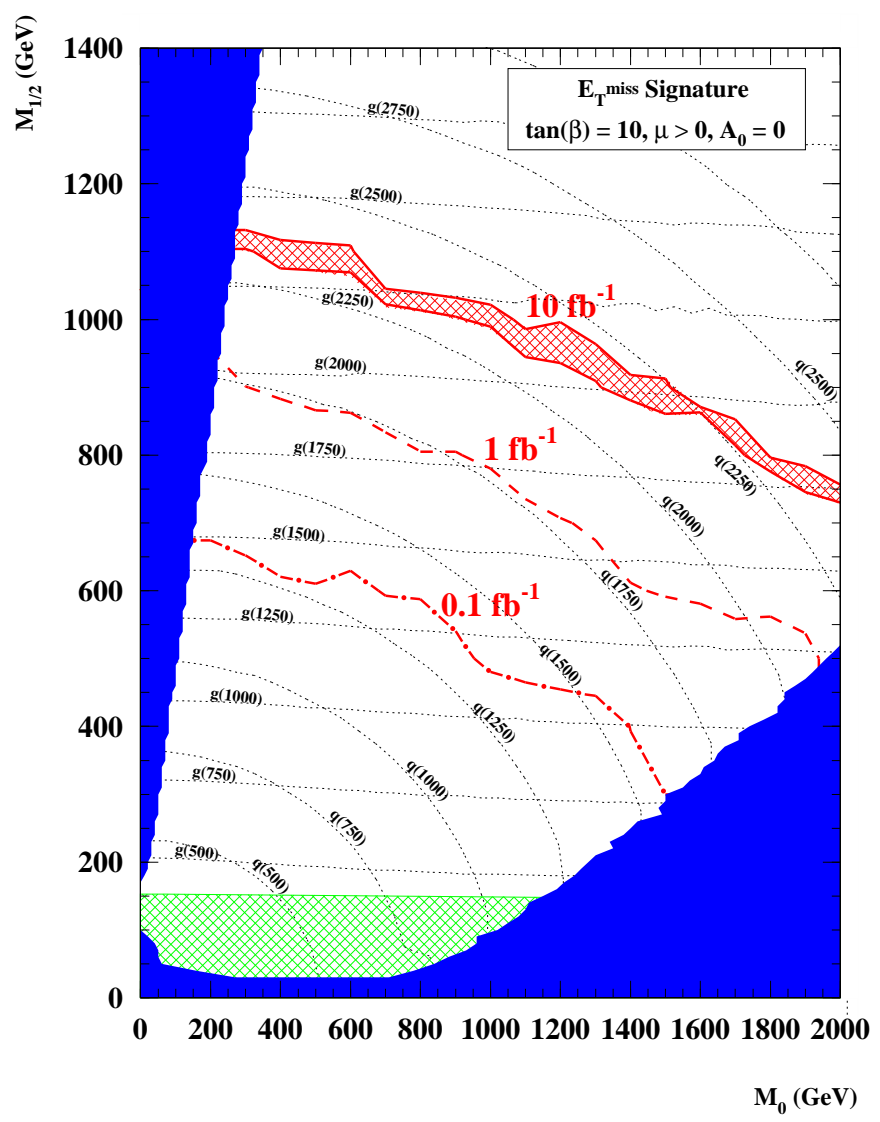

Fig. 11. Discovery curves in the $M_{0}-M_{1 / 2}$ plane from [8]. Shown are lines of constant squark and gluino masses and the discovery potential for given integrated luminosities.

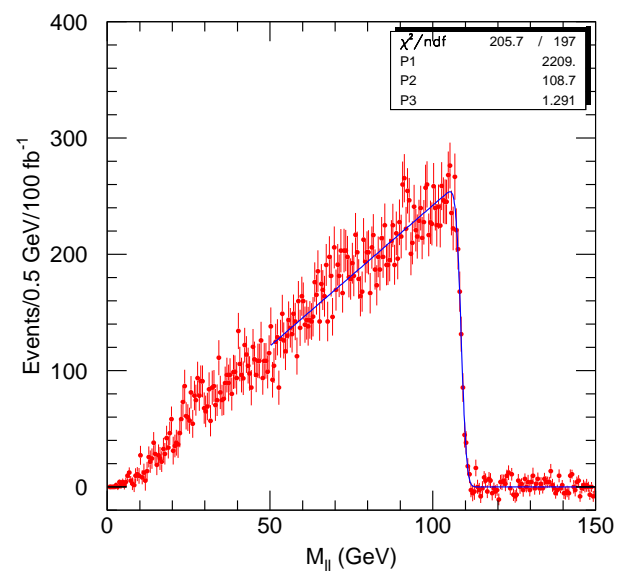

Fig. 12. Invariant mass of lepton pair after background subtraction from like sign lepton pairs. Note that the integrated luminosity used for this plot is $100 \mathrm{pb}^{-1}$. 
the data taken in the first year the position of the edge can be determined with an uncertainty of only a few $\mathrm{GeV}$. Several other decay chains also lead to the determination of mass differences between SUSY Particles. When observing several of these mass differences a more unambiguous signal of SUSY can be established.

Another sign of SUSY is the measurement of the SUSY mass scale $M_{\mathrm{SUSY}}=$ $\min (m(\tilde{q}), m(\tilde{g}))$. When looking at the variable $M_{\mathrm{eff}}=E_{T}^{\mathrm{miss}}+\sum_{i=1}^{4} p_{T}\left(\right.$ jet $\left._{i}\right)$ the peak in this distribution is approximately equal to the SUSY mass scale.

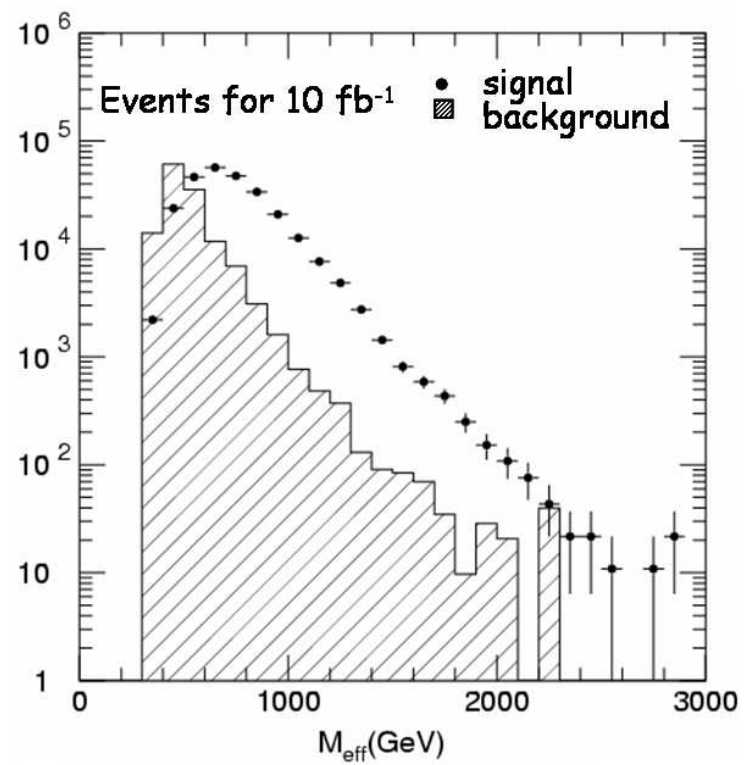

Fig. 13. Distribution of the variable $M_{\mathrm{eff}}=E_{T}^{\mathrm{miss}}+\sum_{i=1}^{4} p_{T}\left(\right.$ jet $\left._{i}\right)$ which is approximately equal to the SUSY mass scale. Shown are the background (histogram) and the signal (solid circles) for squark and gluino masses of around $400 \mathrm{GeV}$.

Figure 13 shows the distribution of $M_{\text {eff }}$ for squark and gluino masses close to the reach of the Tevatron. With the data of the first year a clear signal for SUSY can be established for the overlap region with the Tevatron for gluino and squark masses of around $400 \mathrm{GeV}$. Cuts at $E_{T}\left(\right.$ jet $\left._{1}\right)>80 \mathrm{GeV}$ and $E_{T}^{\text {miss }}>80 \mathrm{GeV}$ can establish this signal and measure the SUSY mass scale for mSUGRA models to approximately 20\%. To make this measurement, low trigger thresholds are necessary as well as a calorimeter coverage with a good hermeticity up to $|\eta|<5$.

\section{Conclusions}

The commissioning of the ATLAS detector is a challenging task. The procedures for commissioning are being developed now, and consist of several steps. They start with the test beam measurements and will end by using known physics signal, like the $\mathrm{Z}$ boson, or straight muons tracks. Due to the large rates of these signals, the alignment of the Inner Detector can be made with a precision better 
than $2 \mu \mathrm{m}$ using muon tracks from only a few days of data taking. A similar data set is able to calibrate the energy scale of the electromagnetic calorimeter using the decay $\mathrm{Z} \rightarrow \mathrm{e}^{+} \mathrm{e}^{-}$.

The main impact from the staging of the detector is that there will be no dedicated B physics trigger during the first year. The impact on other physics analyses is that 10-15\% more integrated luminosity will be needed to achieve the same significance.

Physics results during the first year should include the detection of the Higgs boson over the full mass range. The masses close to the lower mass limit will be the most challenging. If SUSY exists, one or more SUSY Higgs boson will most probably be discovered.

The LHC is a factory for SUSY particles, especially for squarks and gluinos. If these particles exist, a discovery is possible immediately after the start-up of the LHC. The reach for squark masses is already above $2 \mathrm{TeV}$ in the first year.

The data taking in the first year is a very promising time and we all are looking forward to the first proton proton collisions in the year 2007.

\section{References}

1. The ATLAS Collaboration: ATLAS Detector and Physics Performance Technical Design Report, LHCC99-14/15 (1999)

2. B.W. Lee et al.: Phys. Rev. Lett 38 (1977) 883; M. Quiros: Constraints on the Higgs boson properties from the effective potential, hep-ph/9703412; A. Ghinculov and T. Binoth, Acta Phys. Polon. B30 (1999) 99.

3. ALEPH, DELPHI, L3 and OPAL Collaborations: Search for the Standard Model Higgs Boson at LEP, CERN-EP/2003-011 (2003)

4. ALEPH, DELPHI, L3 and OPAL Collaborations, the LEP Electroweak Working Group and the SLD Heavy Flavour Working Group: A Combination of Preliminary Electroweak Measurements and Constraints on the Standard Model, LEPEWWG/2003-01 (2003) and CERN-EP/2002-091 (2002)

5. ZEUS Collaboration; S. Chekanov et al. Eur. Phys. J. C21 (2001) 3, pp 443-471

6. M. Carena et al.: Report of the Higgs Working Group of the Tevatron Run 2 SUSY/Higgs Workshop, hep-ph/0010338

7. S. Asai et al.: Prospects for the Search of a Standard Model Higgs Boson in ATLAS using Vector Boson Fusion, sn-atlas-2003-024 (2003), submitted to Eur. Phys. J. C

8. D.R. Tovey, Inclusive SUSY Searches and Measurements at ATLAS, EPJ Direct, Sect. A-E: 4 (2002) no. CN4, pp.1-24 